\section{Düsseldorfer Hygienepreis}

Anläßlich der 4. Düsseldorfer Hygienetage 1976 gab der Veranstalter, die Henkel KGaA, Düsseldorf, die Stiftung eines Dïsseldorfer Hygienepreises bekannt. Mit diesem Preis sollen hervorragende wissenschaftliche Arbeiten auf dem Gebiet der Hygiene ausgezeichnet werden, die in der Bundesrepublik Deutschland, in Österreich oder in der Schweiz veröffentlicht wurden. Der Preis ist mit 20000 DM dotiert und wird alle zwei Jahre vergeben. Er kann sowohl für eine Arbeit einer Einzelpersönlichkeit als auch einer Gruppe verliehen werden.

Mitglieder des Preistichterkollegiums sind: Prof. Dr. L. $v$. Manger-Koenig (Vorsitz), Prof. Dr. J. Borneff, Prof. Dr. Dr. h. c. A. Mayr, Prof. Dr. H. Schadewaldt, Prof. Dr. H.W. Schlipköter, Prof. Dr. H. Valentin, Dr, B. Werdelmann.

Der Düsseldorfer Hygienepreis soll 1978 auf den 5. Düsseldorfer Hygienetagen (6.-7. April 1978) zum ersten Mal vergeben werden. Berücksichtigt werden Arbeiten, die in den Jahren
1976 und 1977 entstanden sind. Vorschläge müssen bis zum 10. Januar 1978 dem Preisrichterkollegium vorliegen.

Anschrift des Vorsitzenden des Preisrichterkollegiums: Prof. Dr. L, v. Manger-Koenig, Frankenweg 74, 5340 Honnef.

\section{Erratum}

Zu der Arbeit H. M. von Hattingberg: „Pharmakokinetik von Lincomycin und Clindamycin", INFECTION 5 (1977) Supplement 1, Seiten 29-36. Gleichung 15 an Seite 31 muß richtig lauten:

$$
V_{s s}=V_{c} \frac{\sum_{j} \frac{f_{j}}{\gamma_{j}^{2}}}{\left(\sum_{j} \frac{f_{j}}{\gamma_{j}}\right)^{2}}=V_{c} k_{e}^{2} \sum_{j} \frac{f_{j}}{\gamma_{j}^{2}}
$$

\section{Autobac 1-System}

Das erste automatisierte System für die antibakterielle Resistenzbestimmung. Das Autobac 1-System ist eine automatische Methode zur Bestimmung der antibakteriellen Empfindlichkeit von pathogenen Mikroorganismen.

Sie gründet sich auf anerkannte Prinzipien der bakteriellen Resistenzprüfung und wurde besonders deshalb entwickelt, um eine einfache, bequeme und standardisierte Resistenzprüfung zu schaffen, die eine wesentliche Reduktion derjenigen Zeit ermöglicht, die bisher erforderlich ist, um Ergebnisse zu erhalten.

\section{Das System hat folgende Vorteile:}

Hohe Ubereinstimmung in der Bewertung (ca. Durchschnitt 90\% , Bereich 80-100\%) mit den Ergebnissen der Methoden nach Bauer, Kirby, Sherris und Turck innerhalb einer Zeit von 3 bis 5 Stunden nach Selektion der Kolonien

präzise Inokulumstandardisierung

Reproduzierbarkeit der Daten

Reduzierung von Irrtümern/Übertragungsfehlern

erhöhte Effektivität und Bequemlichkeit.

Die photometrische Messung von gestreutem Licht liefert Ergebnisse der antibakteriellen Resistentzbestimmungen für Reinkulturen yon Aerobiern innerhalb einer Zeit yon 3-5 Stunden nach Selektion der Kolonien.
Die Ergebnisse können für die antibiotische Therapie in der Praxis als nützliche Richtlinien einen Tag frïher zur Verfügung stehen.

Autobac 1-Elutionsblättchen, Inokulumstandardisierungslösung, Eugonic-Brühe und Einmalküvetten sind speziell für das System entwickelt worden.

Bei dieser Methode reagieren 12 gleiche Volumina einer photometrisch standardisierten Bakterienkonzentration in einer geschlossenen in 13 Kammern aufgeteilten, optischen Küvette mit einer Serie bis zu 12 verschiedenen Antibiotika. Die 12 Proben: und die nicht gehemmte Kontrolle werden dann gleichzeitig für mindestens 3 Stunden bei $36^{\circ} \mathrm{C}$ inkubiert und durch eine Drehvorrichtung $(220 \mathrm{U} / \mathrm{min}$, Durchmesser $1,9 \mathrm{~cm})$ bewegt.

Wenn nach dieser Zeit in der Kontrolle das Minimum einer Wachstumszunahme von drei Generationen erreicht ist, wird der Prozentsatz der Wachstumshemmung für jedes Antibiotikum automatisch mit dem Photometer gemessen und bewertet (sensibel, mäßig sensibel, resistent).

Ist jedoch das Kontrollwachstum ungenügend, wird die Küvette vom Photometer nicht ausgewertet und muß für eine zusätzliche Inkubationsphase mit Drehbewegung in den Brutschrank zurïckgebracht werden.

Die automatische schriftliche Auswertung der Resistenzbestimmung durch das Photometer erfolgt für eine komplette Kïvette in ca. 15 Sekunden. 